ARTICLE

https://doi.org/10.1038/s41467-020-18977-6

\title{
OPEN
}

\section{Multi-functional flexible 2D carbon nanostructured networks}

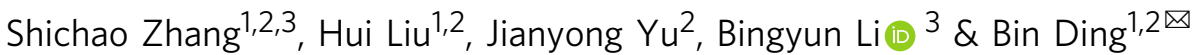

Two-dimensional network-structured carbon nanoscale building blocks, going beyond graphene, are of fundamental importance, and creating such structures and developing their applications have broad implications in environment, electronics and energy. Here, we report a facile route, based on electro-spraying/netting, to self-assemble two-dimensional carbon nanostructured networks on a large scale. Manipulation of the dynamic ejection, deformation and assembly of charged droplets by control of Taylor cone instability and micro-electric field, enables the creation of networks with characteristics combining nanoscale diameters of onedimensional carbon nanotube and lateral infinity of two-dimensional graphene. The macrosized (meter-level) carbon nanostructured networks show extraordinary nanostructural properties, remarkable flexibility (soft polymeric mechanics having hard inorganic matrix), nanoscale-level conductivity, and outstanding performances in distinctly different areas like filters, separators, absorbents, and wearable electrodes, supercapacitors and cells. This work should make possible the innovative design of high-performance, multi-functional carbon nanomaterials for various applications.

\footnotetext{
${ }^{1}$ State Key Laboratory for Modification of Chemical Fibers and Polymer Materials, College of Textiles, Donghua University, 201620 Shanghai, China. ${ }^{2}$ Innovation Center for Textile Science and Technology, Donghua University, 200051 Shanghai, China. ${ }^{3}$ Department of Orthopedics, School of Medicine,

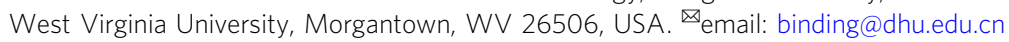


ow-dimensional carbon nanomaterials have significant technological implications for areas in environment, energy, electronics, and healthcare, due to their remarkable mechanical, electronic, optical, and thermal properties ${ }^{1-4}$. Carbon nanoscale building block regimes mainly contain zero-dimensional (0D) nanodots, one-dimensional (1D) nanotubes, twodimensional (2D) graphene, and their derivatives ${ }^{5,6}$. All these blocks share a common structural characteristic of nanoscale sizes, and their extraordinary properties/performances only occur at the nanoscale, rather than at macro-size ${ }^{7-9}$. Various technologies, such as in situ growth, template synthesis, self-assembly, etc. have been proposed and utilized to construct bulk carbon nanomaterials from their isolated blocks ${ }^{10-12}$. Unfortunately, the unique nanoscale properties often disappear or are greatly compromised when assembled into bulk materials. Nature provides a simple yet efficient avenue towards nanomaterial assembly: an ordered fibrous network structure allows materials to show unique properties/functions ${ }^{13-16}$. The leaf veins, spider webs, honeycombs, etc. are vivid cases. They all possess fibrous networks but are structurally robust, high-performance, and multi-functional. Despite extensive efforts, however, existing carbon fibrous nanomaterials mainly depend on deposition of $1 \mathrm{D}$ carbon nanotubes and electrospun nanofibers, in which, the nanotubes suffer from poor continuity (length $<100 \mu \mathrm{m}$ ); for the nanofibers, pseudo-nanoscale $>200 \mathrm{~nm}$ or $\mu \mathrm{m}$ in diameter is inevitable ${ }^{17-21}$. Besides, they both assemble into randomdeposited nonwovens lacking of interior linking, rather than into organized networks. Such intrinsic bottlenecks result in their greatly deteriorated performances. The challenge is, therefore, to create continuous, $2 \mathrm{D}$ network-structured, and macro-sized carbon nanomaterials without compromising their nanoscale properties.

Here, we report a unique electrohydrodynamic methodology, electro-spraying/netting, to generate self-assembled 2D carbon nanostructured networks (N-nets) with well interconnected nanoscale fibers (diameters of $\sim 15 \mathrm{~nm}$, Fig. 1). Our approach allows the dynamic ejection and assembly of droplets from Taylor cone to be tailored, making them assemble into ordered network architectures. The carbon $\mathrm{N}$-nets have both characteristics of nanoscale diameters of $1 \mathrm{D}$ nanotube and lateral infinity of $2 \mathrm{D}$
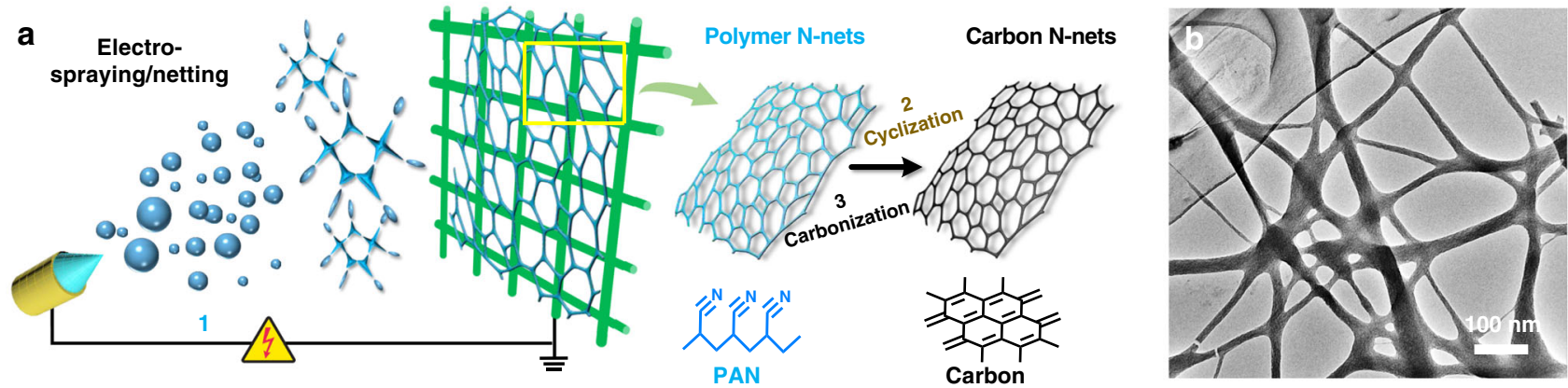

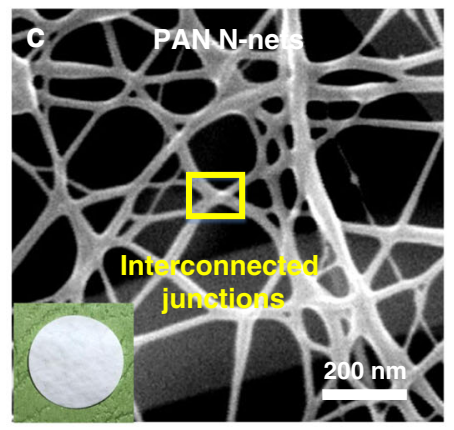

f

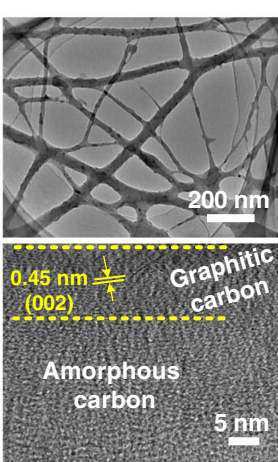

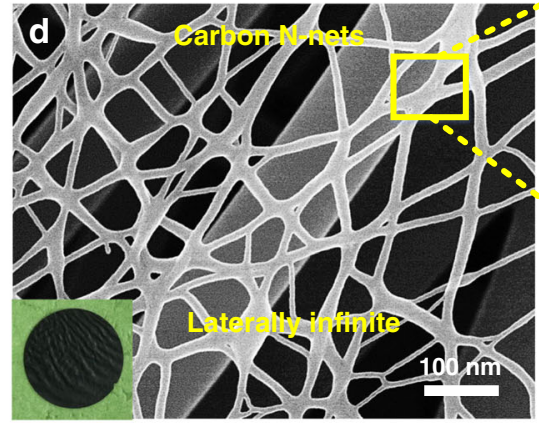

g

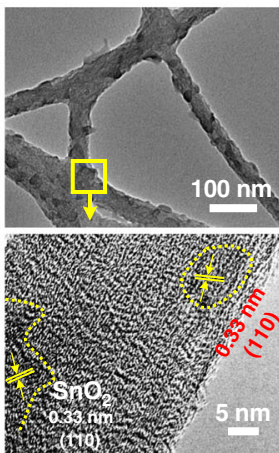

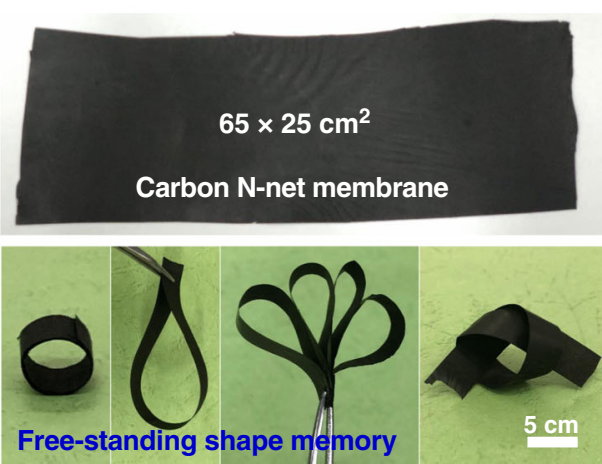
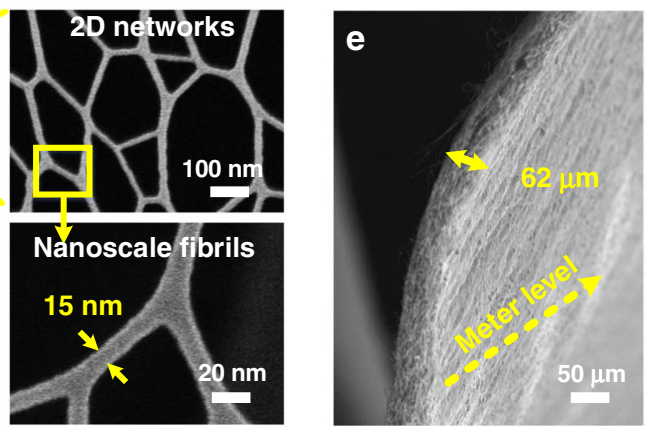

h

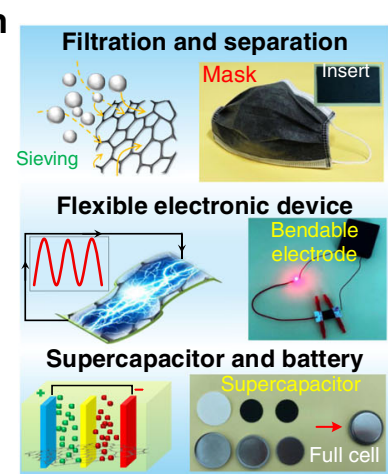

Fig. 1 Processing, architectures, and samples of carbon N-nets. a Schematic showing the synthetic steps of 2D N-nets. (1) Self-assembly of PAN N-nets using electro-spraying/netting. (2) Cyclization, and (3) carbonization to fabricate carbon N-nets. b TEM topography images of the carbon N-nets. SEM images of $\mathbf{c}$ PAN and $\mathbf{d}$ carbon $\mathrm{N}$-nets. The $\mathrm{N}$-nets consist of 1D nanoscale fibrils, with structural characteristics of triangular junctions and laterally infinite 2D networks. e SEM images showing cross-section view of free-standing carbon $\mathrm{N}$-net membranes. $\mathbf{f}$ TEM images of the carbon $\mathrm{N}$-nets and their interior nanostructures. $\mathbf{g}$ A show of large-scale carbon $\mathrm{N}$-net membrane and their flexibility demonstrations. $\mathbf{h}$ Photographs of carbon $\mathrm{N}$-net samples (mask inserts, bendable electrodes, etc.) and related schematics of their various applications. 
graphene. Using these unique $\mathrm{N}$-nets as innovative building blocks, we assembled macro-sized (meter level) carbon nanomaterials that presented nanoscale properties, high flexibility (1050 MPa while softer than napkins), conductivity $\left(920 \mathrm{~S} \mathrm{~cm}^{-1}\right.$, as high as single carbon nanofiber), etc. The same nanomaterials exhibited advanced properties for very different applications like filters, separators, absorbents, wearable electrodes, supercapacitors, and cells.

\section{Results}

Assembly and architectures of carbon N-nets. 2D nanostructured networks (N-nets) were self-assembled using our unique electrospraying/netting technique (Fig. 1a). The formation of N-nets was due to a droplet ejection-deformation-assembly process that underlay Taylor cone instability and differential micro-electric driving. Tailoring of the Taylor cone dynamic and of the microelectric field enabled the highly charged droplets to eject, selfassemble and deform before they phase separated and evolved into a 2D nanofibrous network (Supplementary Discussion). A facile cyclization and calcination of polymeric (polyacrylonitrile, PAN) precursor N-nets led to the generation of carbon N-nets (Supplementary Fig. 1). A closer transmission electron microscopy (TEM) look (Fig. 1b) revealed that the carbon $\mathrm{N}$-nets had both characteristics of nanoscale diameters similar to $1 \mathrm{D}$ carbon nanotube and lateral structures like 2D graphene.

Scanning electron microscopy (SEM) imaging indicated that the PAN N-nets consist of interconnected nanofibers with diameters of 10-40 nm (Supplementary Fig. 2a) and showed 2D topological network structures (Fig. 1c). Besides the unique 2D form, the fiber diameter was true nanoscale, one order of magnitude less than those of conventional nanofibers ${ }^{22,23}$. After oxidization and calcination process, the $2 \mathrm{D} \mathrm{N}$-net structures remained (Supplementary Fig. 3) and their fibers decreased to $5-25 \mathrm{~nm}$ in diameter (Supplementary Fig. 2b), achieving the diameter level of 1D carbon nanotubes (Fig. 1d). Zooming in and out on the carbon N- nets revealed that a continuous, uniform and integrated network formed and was laterally infinite like but superior to $2 \mathrm{D}$ graphene (>meter level compared to $<50 \mu \mathrm{m}$ of graphene in size), as shown in Fig. 1d and Supplementary Fig. 3d. The carbon N-nets are an innovative 2D nanomaterial building block (Fig. 1d, right insets) that is quite different from conventional 1D nanomaterial blocks (nanotube, nanowire, nanorod, etc.) and 2D nanomaterial blocks (graphene, graphitic carbon nitride, transition metal dichalcogenides, etc. $)^{2,24,25}$. Figure 1e displays a free-standing carbon N-net membrane $(\sim 62 \mu \mathrm{m}$ thick) with hierarchical nanostructures (Fig. 1f). Numerous nanoclusters with sizes of 3-12 nm were dispersed in both the carbon matrix and the surface, and caused nanoscale surface roughness (Supplementary Fig. 4). The graphitic carbon layers with inter-planar distance of $0.45 \mathrm{~nm}$ was also observed (Supplementary Fig. 5).

Due to the simplicity of our methodology, great versatility in controlling the precursor polymer category and scaling up the assembly of carbon N-nets was feasible. Figure $1 \mathrm{~g}$ shows a photograph of the carbon N-net membrane with size of $65 \times$ $25 \mathrm{~cm}^{2}$; much larger (at meter level) samples could also be produced (Supplementary Fig. 6). Strikingly, the carbon N-net membranes exhibited robust mechanical strength and excellent softness, and could be folded and bent without any damage, behaving as a sheet of rubber (Fig. 1g bottom). Such remarkable properties, for instance, nanoscale characteristics, 2D network form, robust flexibility, and high conductivity, allow the carbon $\mathrm{N}$-nets to facilitate their widespread applications ranging from filtration and separation to wearable electronic device to supercapacitor and battery ${ }^{26-30}$; some industrial samples (Fig. 1h, such as mask and supercapacitor), using carbon N-nets as core components, were also successfully fabricated.

Synthesis and nanostructures of carbon $\mathrm{N}$-nets. To reveal the self-assembly route and control of N-nets, a possible model was proposed (Fig. 2a). Forces leading to droplet ejection from Taylor

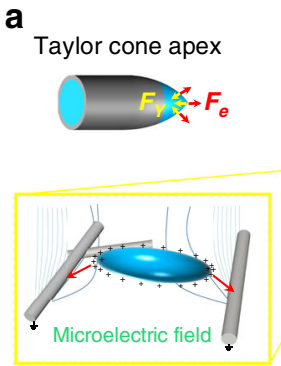

Self-deformation of droplet
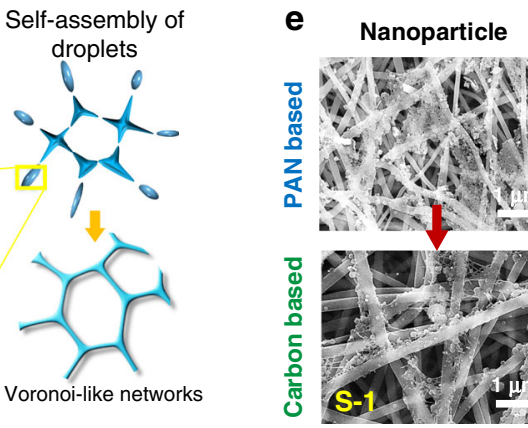

Beaded nanofibrous networks

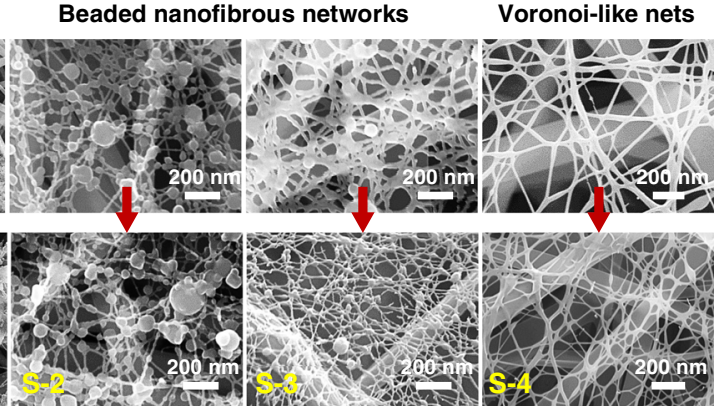

b

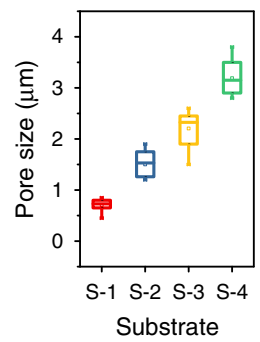

C

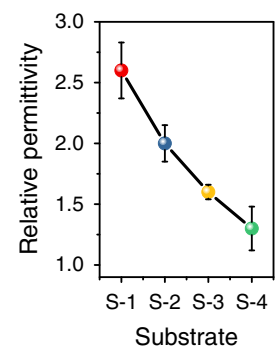

d

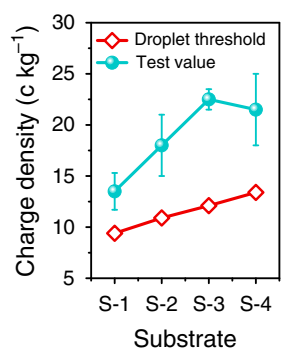

$f$

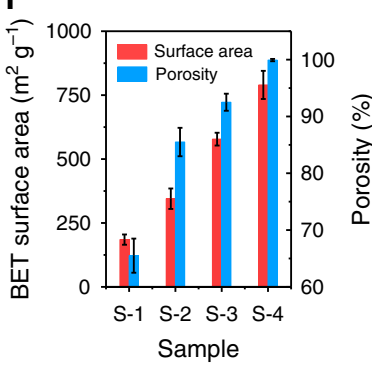

g

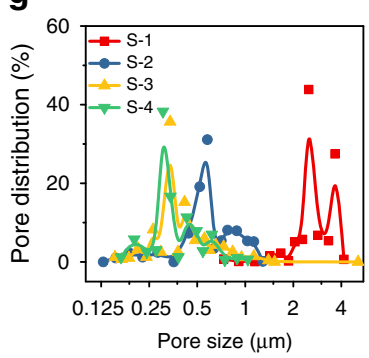

Fig. 2 Origin, synthesis, and nanostructural properties of carbon $\mathbf{N}$-nets. a Schematic illustration of droplet ejection and its deformation-assembly process during electro-spraying/netting. $\mathbf{b}$ Pore size and $\mathbf{c}$ relative permittivity of the utilized substrates, and $\mathbf{d}$ charge density and droplet threshold of the liquids ejected from precursor solutions and collected using these substrates by electro-spraying/netting. S-1-S-4 indicate the four different substrates or

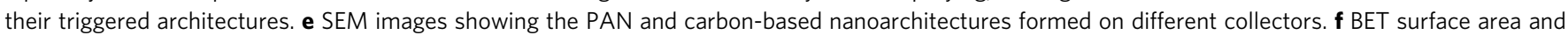
porosity and $\mathbf{g}$ pore size distribution of the membranes consisted of different nanoarchitectures. Base weight in $\mathbf{g}$, $\sim 0.08 \mathrm{~g} \mathrm{~m}^{-2}$. 
cone rely on competing Coulomb repulsion and hydrostatic pressure $^{31,32}$. When charge density of the liquid exceeded the droplet threshold $D_{c},\left(\sqrt{288 \varepsilon \gamma / \delta \rho^{2} D^{3}}\right.$, see Supplementary Notes 1 and 2), the droplets ejected due to Taylor cone instability. Then the levitating cluster of droplets possibly underwent a selfassembly of spatial pattern driven by the Voronoi dissipative effect $^{33,34}$; meanwhile, they self-deformed driven by the differential micro-electric fields, and resulted in the generation of nanofiber networks with Voronoi-like structures. SEM imaging provided evidence supporting this possible formation process (Fig. 2e). Different collectors triggered the assembly of distinct architectures, including nanoparticles, beaded nanofibers, and Voronoi-like networks.

Using electro-spraying/netting technique, manipulation of the surface topography and conductivity of the collectors (Fig. 2b, c, Supplementary Discussion) enabled the creation of polymeric $\mathrm{N}$-nets. Upon increasing the deposition of PAN fibers (from S-4 to S-1, Supplementary Fig. 7a), which used as collectors, their pore size decreased from 3.2 to $0.7 \mu \mathrm{m}$ (Fig. $2 \mathrm{~b}$ ), while permittivity increased from 1.25 to 2.6 (Fig. 2c). An increase in pore size of collectors resulted in the distinct transition from nanoparticles to beaded nanofibers to smooth nanofibers due to enhanced potential gradient of micro-electric fields (Supplementary Note 2). The assembly model (Fig. 2d, Supplementary Methods) indicates that, the gap between the charge density and threshold of the droplets was maximized $\left(10.4 \mathrm{c} \mathrm{kg}^{-1}\right)$ using $\mathrm{S}-3$ collectors, suggesting the highest probability/speed for droplet formation. Obviously, more nanoarchitectures, i.e., beaded nanofibrous networks, were generated on S-3 collectors (Fig. 2e). In addition, the S- 4 collectors induced the creation of Voronoi-like networks because they had both appropriate dielectric property and scaffold pores (Fig. 2a-c).

All carbon-based nanoarchitectures retained their structural characteristics after carbonization from their PAN precursors (Fig. 2e). Compared with the nanoparticles deposited on S-1 substrate $\left(185 \mathrm{~m}^{2} \mathrm{~g}^{-1}\right.$ and $\left.65.5 \%\right)$, both Brunauer-Emmett-Teller (BET)-specific surface area $\left(790 \mathrm{~m}^{2} \mathrm{~g}^{-1}\right)$ and porosity $(99.92 \%)$ of the carbon N-nets on S-4 substrate were significantly enhanced (Fig. 2f), due to their nanoscale fiber diameters (15 nm, Supplementary Fig. 2) and nanoscale roughness (Fig. If and Supplementary Fig. 4). In contrast, most existing electrospun nanofibers typically show BET surface area of $<10 \mathrm{~m}^{2} \mathrm{~g}^{-1}$ and porosity of $50-90 \%$. The emergence of $2 \mathrm{D} \mathrm{N}$-nets in membranes resulted in extremely small pore sizes of 230-320 nm (Fig. $2 \mathrm{~g}$ ) while maintaining a low thickness of $<100 \mathrm{~nm}$; control nanoparticle membrane had pore size of $\sim 3 \mu \mathrm{m}$. These structural properties made our carbon N-nets bear great application potential in high-performance filters, sensors, electronics, and optics.

Properties of carbon N-nets. The carbon N-nets were mechanically robust, along with remarkable surface wettability and electrical conductivity. A single-layered, free-standing carbon $\mathrm{N}$-nets exhibited a tensile strength of $1050 \mathrm{MPa}$ with strain of $\sim 25 \%$, indicating two distinct deformation regimes (Fig. 3a). A closer SEM look (Fig. 3b top) at the stretching process of $0-30 \% \varepsilon$, indicated that the carbon N-nets effectively transferred and dissipated loads. This unique deformation involved net deformation and fibril aligning (Fig. 3b bottom). Tensile stress firstly restricted the nanofibrils in $\mathrm{N}$-nets to align increasingly in a plane along the stretching direction, and led to an undamaged net-shape change, yielding an elastic behavior $(0-10 \% \varepsilon)$. Further fibril aligning within $13-20 \% \varepsilon$ allowed for rapidly increased stresses and nonlinear deformation until fragile $(>30 \% \varepsilon)$. Moreover, the carbon $\mathrm{N}$-nets showed a bending rigidity of $8.5 \mathrm{mN}$, which was only $1 / 10$ of polyethylene nonwovens $(110 \mathrm{mN})$ or electrospun carbon nanofiber membranes $(85.5 \mathrm{mN})$. Such performance means that the carbon N-nets possessed soft polymeric mechanical properties while having hard inorganic matrix. The carbon N-nets even had smaller bending rigidity than that $(28.5$ $\mathrm{mN}$ ) of tissue paper, suggesting superior softness thereby making it possible to be used as wearable conductive nanomaterials for emerging energy and electronics applications ${ }^{35}$.

The flexibility of the carbon N-nets was further demonstrated by their cycling compression-release evaluation (Fig. 3d). A movie (Supplementary Movie 1) demonstrated that a circular ring made of free-standing carbon N-nets $\left(1.5 \times 10 \mathrm{~cm}^{2}\right)$ immediately recovered its original shape when lifting a weight of $200 \mathrm{~g}$ (300 times heavier than itself), showing striking shape-memory performance ${ }^{36}$. We easily tied a knot using such a carbon $\mathrm{N}$ net membrane. To gain insight into the remarkable flexibility, the hierarchical structures of carbon N-net membranes during deformation were studied by in situ SEM (Fig. 3d bottom). At the top level, the membrane was organized in a lamellar-mesh geometry with sponge-like structure to maximize the stress absorption (thickness $h$ increased twice after bending). At the meta-scale level, the $2 \mathrm{D}$ interconnected networks, with resilient and restorable deformation capability $(20 \%$ restorable strain, Fig. 3b), can transfer loading strain with minimum network bending. At the low level, a single carbon nanofiber can bear a large bending deformation without generating any cracks, due to the lubrication matrix structures consisting of amorphous carbon, ordered graphitic carbon and dispersed $\mathrm{SnO}_{2}$ nanoclusters (Fig. 1g). Carbon N-nets showed superoleophilicity with an oil contact angle (OCA) of $<3^{\circ}$, which was negligible. A high-speed camera visualized their dynamic wetting behavior (Fig. $3 \mathrm{e}$ and Supplementary Fig. 8). On the surface, the oil droplet $(3 \mu \mathrm{l}$, ethylene glycol) spread out quickly $(1.8 \mathrm{~s})$ due to the nanocapillary effect ${ }^{37}$. However, the carbon $\mathrm{N}$-nets were superhydrophobic with water contact angles (WCA) of $>152^{\circ}$, and they exhibited extremely low water adhesion (Supplementary Fig. 8), revealing self-cleaning capability of wearable electronics ${ }^{38}$.

Despite striking electrical conductivity of isolated nanoscale blocks of carbon materials (such as nanotube and graphene), bringing such remarkable properties to their macroscopic bulks is nearly impossible ${ }^{39-42}$. This challenge is due to the lack of the effective interconnection between isolated blocks. Here, our freestanding carbon $\mathrm{N}$-nets can function as a macroscopic graphene sheet (>meter level). The well graphitized carbon $\mathrm{N}$-nets presented high conductivity ( $920 \mathrm{~S} \mathrm{~cm}^{-1}$, Fig. 3f), $>5$ times of those of CNF bulks $\left(1-130 \mathrm{~S} \mathrm{~cm}^{-1}\right)$ and CNT bulks $\left(15-85 \mathrm{~S} \mathrm{~cm}^{-1}\right)$ and similar to those of isolated CNF building blocks $\left(200-1500 \mathrm{~S} \mathrm{~cm}^{-1}\right)$. This was because that the integrated $2 \mathrm{D}$ network resulted in a more steadily and continuously conductive path compared to conventional isolated blocks 39,43 . Our carbon $\mathrm{N}$-nets also showed excellent fatigue resistance (Fig. 3g, h and Supplementary Fig. 5). After 10,000 cycle fatigue tests with a completely folded angle of $180^{\circ}$ (Fig. $3 \mathrm{~g}$ and Supplementary Movie 2), the conductivity and stiffness of the carbon $\mathrm{N}$-net membranes (pyrolysis at $1000^{\circ} \mathrm{C}$ ) remained almost unchanged, maintaining high levels of $\sim 900 \mathrm{~S} \mathrm{~cm}^{-1}$ and $8.8 \mathrm{mN}$ (Fig. 3h), indicating promising application for flexible metacomposites $^{44-46}$.

Multifunctionality of carbon N-nets. In considering the small pore size, 2D network structure and high porosity (Figs. 1d, 2f, g), applications in filtration and separation by the carbon $\mathrm{N}$-nets were possible. Particulate matter (PM) pollution in air has raised serious concerns for public health ${ }^{47-49}$. However, most air filters are heavy, bulky, and show inevitable compromise between removal efficiency and air resistance. Our ultrathin carbon N-nets provided an innovative material for high-efficiency and low- 

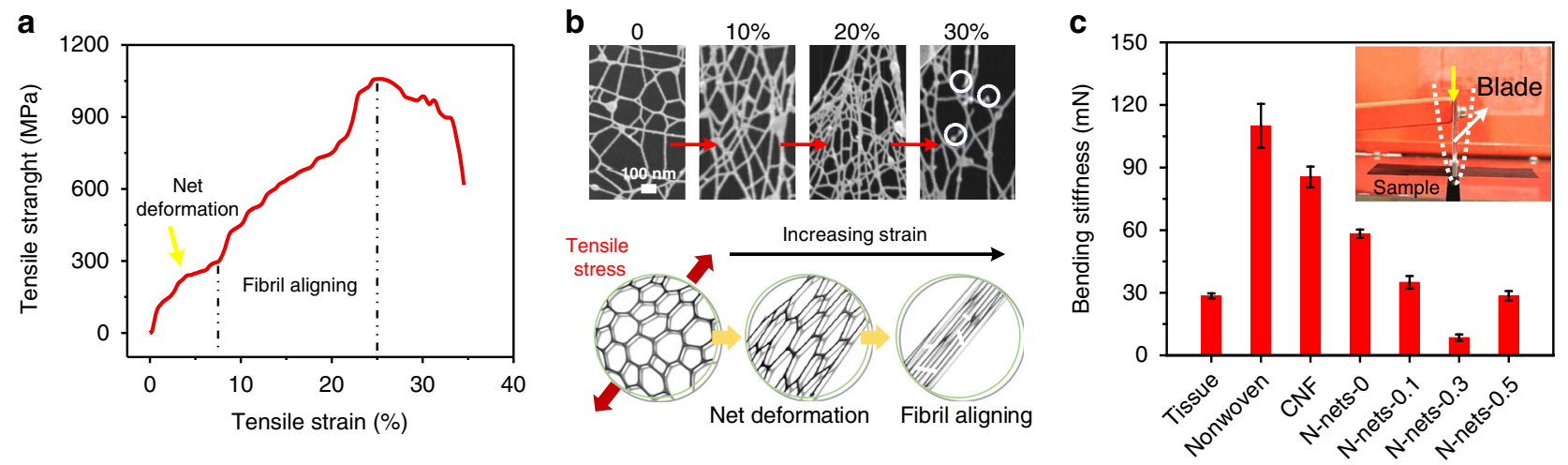

d

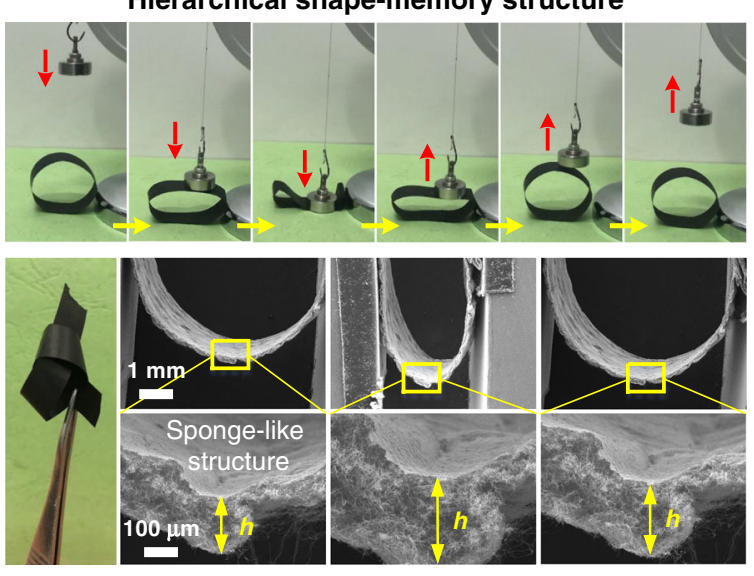

e

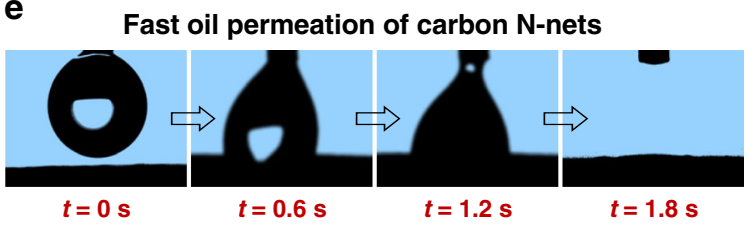

$\mathbf{f}$

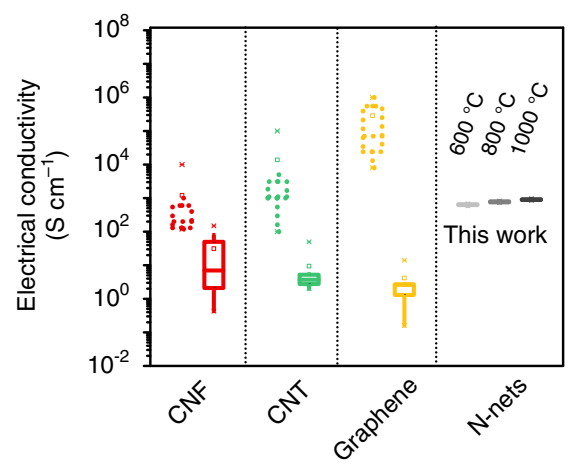

g

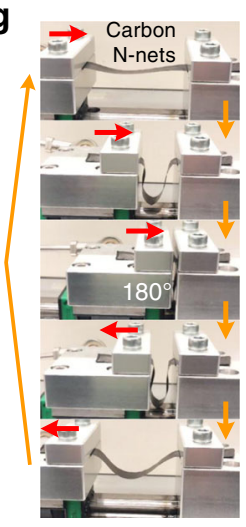

$\mathbf{h}$

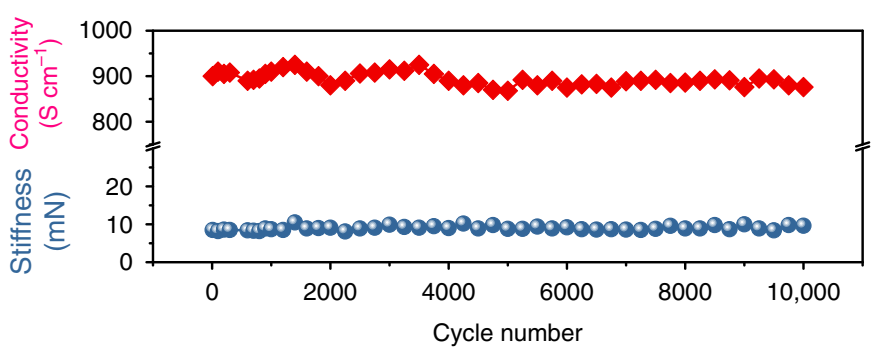

Fig. 3 Mechanical properties, surface wettability, and electrical conductivity of the carbon $\mathbf{N}$-nets. a Microtensile-strain curve of the free-standing carbon N-nets. b SEM images (top) and schematic evolution (bottom) of carbon N-nets during a continuous stretching process. c Bending rigidities of the carbon $\mathrm{N}$-nets and selected soft materials. $\mathrm{N}$-nets-X indicates carbon $\mathrm{N}$-nets prepared from PAN solutions with $\mathrm{X}$ wt $\% \mathrm{SnCl}$. Base weight of $\mathrm{N}$-nets, $\sim 300 \mathrm{mg} \mathrm{m}^{-2}$. d A set of real-time images showing the flexibility of carbon $\mathrm{N}$-nets (top). SEM observations under cycled compression and release, focusing on a piece of free-standing carbon $\mathrm{N}$-nets (bottom). e Optical images of dynamic oil spreading on the surface of carbon $\mathrm{N}$-net membranes. $\mathbf{f}$ Electrical conductivities of typical carbon nanomaterials (carbon nanofiber or CNF, carbon nanotube or CNT, graphene, and carbon N-nets) in different forms. The solid data boxes indicate assembled bulks, and the dashed data boxes show their isolated building blocks. $\mathbf{g}$ Photographs showing the cycle fatigue test of the carbon $\mathrm{N}$-nets with bending angle of $180^{\circ}$ and $\mathbf{h}$ their cycling electrical and mechanical performance within 10,000 cycles.

resistance air filtration, which was used as filter inset for N100 mask (Fig. 4a inset). Besides $\mathrm{PM}_{2.5}$, the carbon $\mathrm{N}$-net filters demonstrated outstanding efficacy for the sternest $\mathrm{PM}_{0.3}$ (PM concentration of 300,000-500,000) removal (Fig. 4a). Carbon Nnet filters with $45 \mathrm{mg} \mathrm{m}^{-2}$ showed efficiency $99.533 \%$ for $\mathrm{PM}_{03}$ removal and $99.925 \%$ for $\mathrm{PM}_{2.5}$ removal at pressure drop of $15 \mathrm{~Pa}$. A $99.992 \%$ efficiency of $\mathrm{PM}_{0.3}$ removal and $99.999 \%$ efficiency (ultralow penetration air level) of $\mathrm{PM}_{2.5}$ removal by $\mathrm{N}$-net filters were achieved at a pressure drop of $65 \mathrm{~Pa}$, exhibiting a higher quality factor (even $0.35 \mathrm{~Pa}^{-1}$, Supplementary Fig. 9) and better performance than most existing filters. With increasing PM concentration to 20 million, the PM removal capability of the carbon N-nets was slightly decreased, for instance, from 99.992 to 99.985\% for $\mathrm{PM}_{0.3}$ removal (Supplementary Fig. 10). The air resistance was only $0.06 \%$ of the atmospheric pressure, which was negligible. In addition, their base weights were about one order of magnitude less than those of conventional nanofiber filters (2-30 $\mathrm{g} \mathrm{m}^{-2}$ ), and were negligible compared to conventional microfiber filters (usually $>100 \mathrm{~g} \mathrm{~m}^{-2}$ ). We believe that the robust removal efficacy of carbon $\mathrm{N}$-nets rely on small pore size and high interface energy, and their low air resistance is due to high porosity and the slip-effect for airflow from high Knudsen number of 8.8 (Supplementary Table 1).

Due to the numerous channels stemmed from the highly porous architecture and extended surface area (Fig. 2f), water treatment (liquid separation and dye absorption, Fig. 4b, c) by carbon N-nets was expected. Solely by gravity-driven, the carbon $\mathrm{N}$-net membrane $\left(320 \mathrm{mg} \mathrm{m}^{-2}\right.$ ) exhibited rejection efficiency of $>99.90 \%$ and permeate flux of $3200 \mathrm{l} \mathrm{m}^{-2} \mathrm{~h}^{-1}$ (Supplementary Fig. 11). Such fluxes were $>10$ times higher than those of microfiltration and ultrafiltration 
a

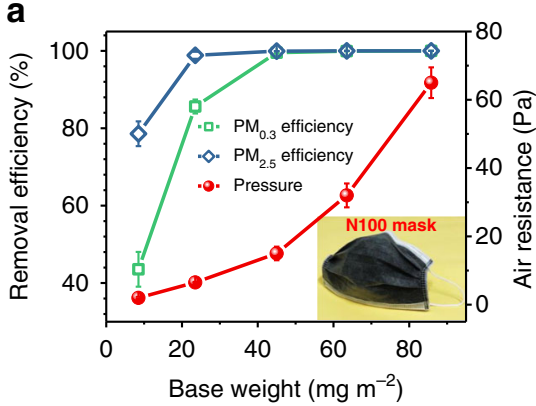

d

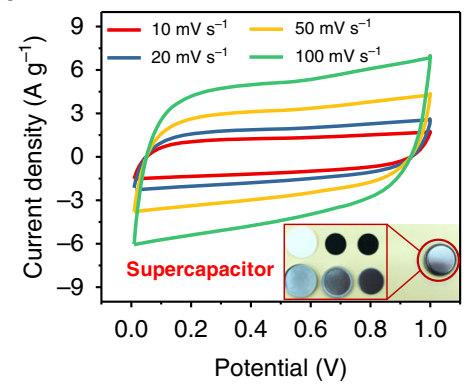

b

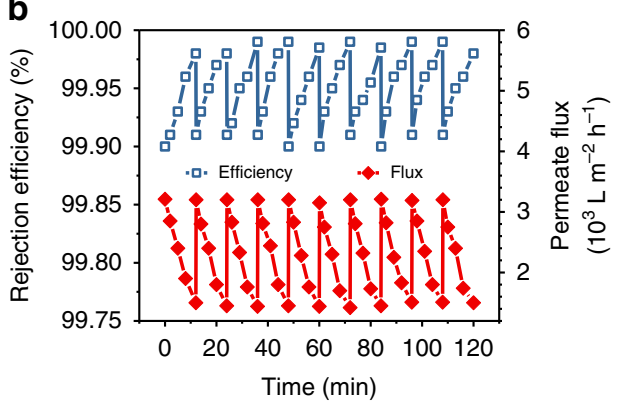

C

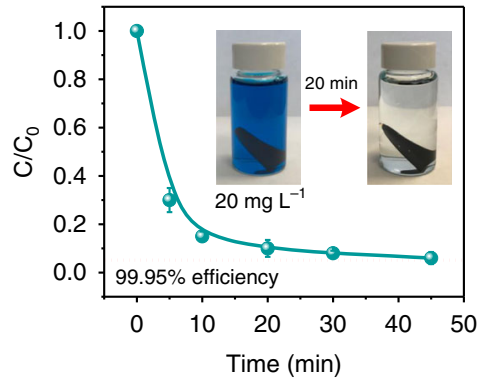

e

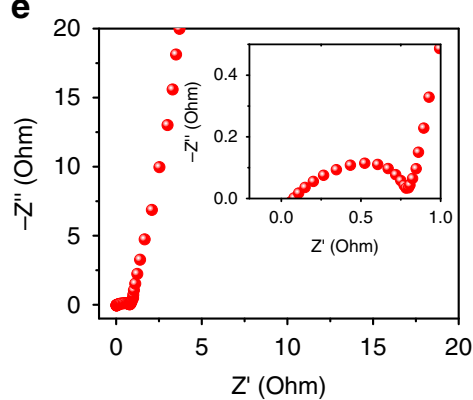

f

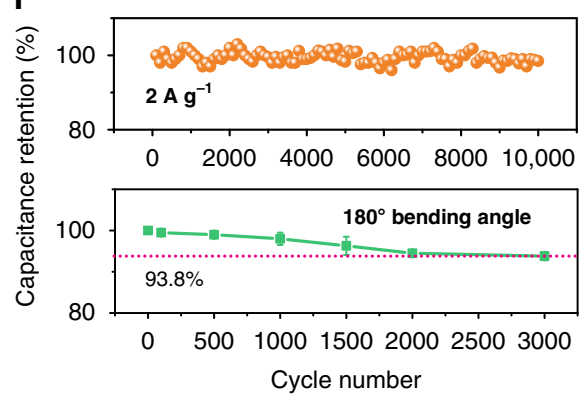

Fig. 4 Multifunctionality of combining the air filtration, liquid separation, dye absorption, and electrochemical applications. a $P M_{0.3}$ and $P M_{2.5}$ filtration efficiency and air resistance of the carbon $\mathrm{N}$-nets with various base weights. Airflow velocity, $5.33 \mathrm{~cm} \mathrm{~s}^{-1}$. Inset of $\mathbf{a}$ is the prepared industrial sample of N100 mask using carbon $\mathrm{N}$-nets as core inset. $\mathbf{b}$ Cycling liquid separation performance of the carbon $\mathrm{N}$-nets driven by gravity. Base weight in $\mathbf{b}, \sim 320 \mathrm{mg} \mathrm{m}^{-2}$. $\mathbf{c}$ Dye absorption performance of the carbon $\mathrm{N}$-nets. Inset of $\mathbf{c}$ contains photos showing the dye solutions before and after absorption. $\mathbf{d} \mathrm{CV}$ curves of the carbon $\mathrm{N}$-nets at different scan rates. Inset of $\mathbf{d}$ is the fabricated supercapacitor using carbon $\mathrm{N}$-nets as electrode. e Nyquist plots of the carbon $\mathrm{N}$-nets in the frequency range of $10-10^{5} \mathrm{~Hz}$. $\mathbf{f}$ Cycling performance of carbon $\mathrm{N}$-nets in supercapacitors at a current density of $2 \mathrm{Ag}^{-1}$ (top) and after fatigue test with a bending angle of $180^{\circ}$ (bottom).

membranes with similar efficiency levels. With increasing separation time, the rejection efficiency increased from 99.90 to $99.99 \%$, and the permeate flux reduced from 3200 to $1520 \mathrm{l} \mathrm{m}^{-2} \mathrm{~h}^{-1}$ (Fig. 4b). This result suggested that the carbon $\mathrm{N}$-nets functioned in a surface filtration manner, leading to the generation of filter cakes. Therefore, after a facile cleaning with water, their separation efficacy was recovered completely (even after 10 cycles, Fig. 4b), a characteristic that has rarely been achieved in present separation membranes but is desirable for cross-flow filtration. The carbon N-nets also could be used as a promising adsorbent for organic pollutant absorption ${ }^{50}$; a small piece of carbon $\mathrm{N}$-net membrane $(3 \mathrm{mg}$ ) could adsorb most of methylene blue, a pollutant model $\left(20 \mathrm{mgl}^{-1}, 30 \mathrm{ml}\right)$, in $20 \mathrm{~min}$, and achieved $99.95 \%$ efficiency within $45 \mathrm{~min}$ (Fig. 4c). Such a high adsorption of $200 \mathrm{mg} \mathrm{g}^{-1}$ could be attributed to their hierarchical roughness (Fig. 1f) and enhanced surface area (Fig. 2f).

The carbon $\mathrm{N}$-nets offered robust conductivity for energy storage and transfer (Fig. 3f), besides, their flexible property also allowed them to be used for wearable electronics (Fig. $3 \mathrm{~g}$, $\mathrm{h}$ and Supplementary Movie 2). The direct use of free-standing carbon Nnets as electrodes avoided the requirement of loading binders or conductive additives that restrained fast discharge/charge ${ }^{51}$. Here, we assembled all carbon supercapacitors using carbon N-net symmetric electrodes (Fig. 4d). The obtained supercapacitor showed a typical rectangular cyclic voltammograms (CV) curve with redox peaks. This result indicated that the electrical-doublelayer capacitance and pseudocapacitance functioned together, which might be arisen from the carbon matrix and doped $\mathrm{SnO}_{2}$, respectively. Increasing scan rate $\left(10-100 \mathrm{mV} \mathrm{s}^{-1}\right)$ did not change the CV curve shape, indicating the high rate capability. The carbon $\mathrm{N}$-net supercapacitor showed a specific capacitance of $275 \mathrm{~F} \mathrm{~g}^{-1}$ at the rate of $10 \mathrm{mV} \mathrm{s}^{-1}$, achieving remarkable performance like the reported hierarchical activated carbon supercapacitors ${ }^{52}$.
Figure $4 \mathrm{e}$ and Supplementary Fig. 12 present the electrochemical impedance data. The straight line of Nyquist plot $\left(10-10^{5} \mathrm{~Hz}\right)$ of the carbon $\mathrm{N}$-nets in the low frequency region indicated high ionic accessibility, with an equivalent series resistance of $0.08 \Omega$, suggesting a low internal resistance of the whole cell. In contrast to carbon nanofibers ( $29 \Omega$, Supplementary Figs. $7 \mathrm{~b}$ and 12 ), the charge resistance transfer $\mathrm{R}_{\mathrm{CT}}$ of carbon N-nets was only $0.78 \Omega$, demonstrating striking transmission of electrons and ions. The galvanostatic charge/discharge (0.5-4 $\mathrm{A} \mathrm{g}^{-1}$, Supplementary Fig. 13) of carbon N-net supercapacitors also showed a symmetrical triangular shape with slight nonlinearities and a short chargedischarge time, confirming its high reversibility. The cycling performance at $2 \mathrm{Ag}^{-1}$ (Fig. $4 \mathrm{f}$ top) indicated that the $\mathrm{N}$-net supercapacitors retained $>96 \%$ of the initial capacitance after 10,000 cycles. More strikingly, the used carbon N-net electrodes maintained $93.8 \%$ of their original capacitance even after being bent to the angle $>180^{\circ} 3000$ times (Fig. $4 \mathrm{f}$ bottom), revealing their intriguing application potential for wearable electronics.

\section{Discussion}

The synthesis of carbon N-nets provides an innovative 2D building block, which is quite different from nanomaterial $1 \mathrm{D}$ blocks (nanotube, nanowire, etc.) and 2D blocks (graphene, transition metal dichalcogenides, etc.), to develop high-performance carbon nanomaterials. The carbon $\mathrm{N}$-nets showed ordered nanofibrous networks and were laterally infinite, which enabled them to be used directly in pseudo-3D macroscopic form or as core fillers for high-performance nanocomposites ${ }^{53,54}$. Considering the ease of scalable synthesis of $2 \mathrm{D} \mathrm{N}$-nets using electro-spraying/netting, our findings pave the way for new types of carbon-based N-nets from various polymeric precursors (such as cellulose, poly(amic acid), 
polyimide, etc.) for use in diverse applications. Moreover, similar to the carbonization, a variety of oxide ceramic N-nets could be processed by combining with a sol-gel process, and would find widespread use in energy and electronics. In addition, various functions (e.g., photoactive, antimicrobial, catalytic, and magnetic properties), desirable for applications like catalysis, tissue engineering, drug delivery, etc., could be easily endowed with composite $\mathrm{N}$-net materials simply by incorporating functional fillers in precursor solutions or by post-coating on the carbon N-net scaffolds.

In concert, our studies present an innovative self-assembly strategy for the scalable synthesis of carbon $\mathrm{N}$-nets with 2D network structures by unique electro-spraying/netting technique. Such pure nanoarchitectured fibrous networks show the integrated properties of nanoscale diameters $(5-25 \mathrm{~nm})$ like $1 \mathrm{D}$ carbon nanotube and lateral infinity like 2D graphene. Our data suggest that these carbon $\mathrm{N}$-nets demonstrate various remarkable properties, such as nanostructural characteristics, robust mechanical flexibility, unique surface wettability and superior electric conductivity. As expected, the carbon N-nets yielded excellent performances towards multifunctional applications of air filtration, liquid separation, dye absorption, and electrochemical applications. This work paves the way for creating such exceptional carbon nanomaterials and opens up numerous opportunities for applications in environment, energy, electronics, catalysis, and tissue engineering.

\section{Methods}

Fabrication of carbon $\mathbf{N}$-nets membranes. The electro-spraying/netting process was performed using a conventional DXSN spinning machine (SOF Nanotechnology Co., Ltd., China). The detailed fabrication process and solution properties are presented in Supplementary Methods and Supplementary Table 2. The used precursor solutions were prepared by dissolving $3 \mathrm{wt} \%$ PAN $\left(M_{\mathrm{w}}=50,000\right)$ in $\mathrm{N}, \mathrm{N}$-dimethyformamide/stannous chloride $\left(\mathrm{DMF} / \mathrm{SnCl}_{2}\right)$ solvents. The $\mathrm{SnCl}_{2}$ concentrations in solutions were tuned to $0,0.1,0.3$, and $0.5 \mathrm{wt} \%$. The solutions were ejected with feed rate of $0.1 \mathrm{ml} \mathrm{h}^{-1}$ to form levitating clusters of charged droplets powered by a DC voltage of $30 \mathrm{kV}$ with distance of $15 \mathrm{~cm}$. Designed substrates, nanofiber membranes electrospun from $13 \mathrm{wt} \%$ PAN solutions with different spinning durations, were used as collectors to collect the resultant architectures. After vacuum drying $\left(50^{\circ} \mathrm{C}\right.$ for $\left.1 \mathrm{~h}\right)$, the as-prepared PAN N-nets were stabilized by oxidization in air at $280^{\circ} \mathrm{C}$ for $2 \mathrm{~h}$. Then carbonization process protected in $99.999 \% \mathrm{~N}_{2}$ flow for $2 \mathrm{~h}$ was utilized to fabricate carbon $\mathrm{N}$-nets. The temperatures were 600,800 and $1000{ }^{\circ} \mathrm{C}$ with a heating rate of $2^{\circ} \mathrm{C} \mathrm{min}-1$.

Characterization. Solution properties including viscosity, conductivity and surface tension, and the charge density of the droplets ejected from metal sprayers during electro-spraying/netting were examined as depicted in Supplementary Methods. The morphologies and structures of the nanostructured architectures were characterized using SEM (Hitachi S-4800, precoated with gold for $150 \mathrm{~s}$ ) and TEM (JEM-2100F). The thickness and base weight of the carbon N-nets were measured using Labthink thickness gauge (CHYC2, readability of $0.1 \mu \mathrm{m})$ and Mettler Toledo Micro balance (AT-20, readability of $2 \mu \mathrm{g}$ ), respectively. The specific surface area and pore structure of the carbon $\mathrm{N}$-nets were characterized by BET

ASAP2020 surface area analyzer (Micromeritics Co., USA) and CFP-1100AI capillary flow porometer (Porous Materials Inc., USA). The surface wettability of the carbon N-nets was examined using a contact angle goniometer (SL200B), and their mechanical property data were collected using single-nanofiber mechanical tester (FSF001.1, precision of $1 \mu \mathrm{N}$ ), programmable sliding table and softness tester (RRY-1000). The electrical conductivity of the carbon N-nets was determined with multi-functional four-point probe apparatus (ST-2258C). The filtration performance, such as removal efficiency and air resistance of the carbon $\mathrm{N}$-net-based masks was measured using LZC-G filter equipment (Huada Filter Technology Co., Ltd., China), the testing details are shown in Supplementary Methods. The liquid separation and dye absorption tests of the carbon $\mathrm{N}$-nets were carried out using $100 \mathrm{ppm} \mathrm{TiO}_{2}$ nanoparticle suspension and $20 \mathrm{mg} \mathrm{l}^{-1} \mathrm{MB}$ solution, respectively. The dye and nanoparticle concentrations in solutions were evaluated using UV-vis spectrometer (PG 2000+) with an integrating sphere. The electrochemical measurements were performed in a two-electrode system using electrochemical workstation (Biologic VMP3). CR2025-type coin cells, which used carbon N-net membranes as self-supported electrodes, were utilized and detailed in Supplementary Methods.

\section{Data availability}

The experimental data that support the findings of this study are available from the corresponding author upon reasonable request.
Received: 14 February 2020; Accepted: 23 September 2020; Published online: 12 October 2020

\section{References}

1. Fitzgibbons, T. C. et al. Benzene-derived carbon nanothreads. Nat. Mater. 14, 43-47 (2015).

2. De Volder, M. F., Tawfick, S. H., Baughman, R. H. \& Hart, A. J. Carbon nanotubes: present and future commercial applications. Science 339, 535-539 (2013)

3. Jariwala, D., Sangwan, V. K., Lauhon, L. J., Marks, T. J. \& Hersam, M. C. Carbon nanomaterials for electronics, optoelectronics, photovoltaics, and sensing. Chem. Soc. Rev. 42, 2824-2860 (2013).

4. Nidamanuri, N., Li, Y., Li, Q. \& Dong, M. Graphene and graphene oxidebased membranes for gas separation. Eng. Sci. 9, 3-16 (2020).

5. Pachfule, P., Shinde, D., Majumder, M. \& Xu, Q. Fabrication of carbon nanorods and graphene nanoribbons from a metal-organic framework. Nat. Chem. 8, 718 (2016).

6. Ahn, E. C., Wong, H. S. P. \& Pop, E. Carbon nanomaterials for non-volatile memories. Nat. Rev. Mater. 3, 18009 (2018).

7. Yao, Y. et al. Carbon welding by ultrafast joule heating. Nano Lett. 16, 7282-7289 (2016).

8. Behabtu, N. et al. Strong, light, multifunctional fibers of carbon nanotubes with ultrahigh conductivity. Science 339, 182-186 (2013).

9. Whitesides, G. M. The 'right' size in nanobiotechnology. Nat. Biotechnol. 21 , 1161-1165 (2003)

10. Park, H. et al. High-density integration of carbon nanotubes via chemical selfassembly. Nat. Nanotechnol. 7, 787-791 (2012).

11. Wang, J. et al. Hierarchical porous carbons with layer-by-layer motif architectures from confined soft-template self-assembly in layered materials. Nat. Commun. 8, 15717 (2017).

12. Chen, K. et al. Printed carbon nanotube electronics and sensor systems. $A d v$ Mater. 28, 4397-4414 (2016).

13. Shao, Z. \& Vollrath, F. Materials: surprising strength of silkworm silk. Nature 418, 741 (2002).

14. Steven, E. et al. Carbon nanotubes on a spider silk scaffold. Nat. Commun. 4, 2435 (2013)

15. Cranford, S. W., Tarakanova, A., Pugno, N. M. \& Buehler, M. J. Nonlinear material behaviour of spider silk yields robust webs. Nature 482, 72 (2012).

16. Wegst, U. G., Bai, H., Saiz, E., Tomsia, A. P. \& Ritchie, R. O. Bioinspired structural materials. Nat. Mater. 14, 23-26 (2015).

17. Segawa, Y., Ito, H. \& Itami, K. Structurally uniform and atomically precise carbon nanostructures. Nat. Rev. Mater. 1, 15002 (2016).

18. Zhang, S. et al. Direct electronetting of high-performance membranes based on self-assembled 2D nanoarchitectured networks. Nat. Commun. 10, 1458 (2019).

19. Chen, B. et al. Length effect of carbon nanotubes on the strengthening mechanisms in metal matrix composites. Acta Mater. 140, 317-325 (2017).

20. Zhang, B., Kang, F., Tarascon, J. M. \& Kim, J. K. Recent advances in electrospun carbon nanofibers and their application in electrochemical energy storage. Prog. Mater. Sci. 76, 319-380 (2016).

21. Inagaki, M., Yang, Y. \& Kang, F. Carbon nanofibers prepared via electrospinning. Adv. Mater. 24, 2547-2566 (2012).

22. Li, D. \& Xia, Y. Electrospinning of nanofibers: reinventing the wheel? Adv. Mater. 16, 1151-1170 (2004).

23. Nataraj, S., Yang, K. \& Aminabhavi, T. Polyacrylonitrile-based nanofibers-a state-of-the-art review. Prog. Polym. Sci. 37, 487-513 (2012).

24. El-Kady, M. F., Shao, Y. \& Kaner, R. B. Graphene for batteries, supercapacitors and beyond. Nat. Rev. Mater. 1, 16033 (2016).

25. Chen, T. \& Dai, L. Carbon nanomaterials for high-performance supercapacitors. Mater. Today 16, 272-280 (2013).

26. Hafez, A. M., Sheng, J., Cao, D., Chen, Y. \& Zhu, H. Flexible lithium metal anode featuring ultrahigh current density stability with uniform deposition and dissolution. ES Energy Environ. 5, 85-93 (2019).

27. Shi, S. et al. Robust flexible poly(amidoxime) porous network membranes for highly efficient uranium extraction from seawater. Nano Energy 71, 104629 (2020).

28. Cai, J. et al. Robust construction of flexible bacterial cellulose@Ni(OH $)_{2}$ paper: toward high capacitance and sensitive $\mathrm{H}_{2} \mathrm{O}_{2}$ detection. Eng. Sci. 5, 21-29 (2019).

29. Luo, Q. et al. All-carbon-electrode-based endurable flexible perovskite solar cells. Adv. Funct. Mater. 28, 1706777 (2018).

30. Liu, Y. et al. High-performance coaxial wire-shaped supercapacitors using ionogel electrolyte toward sustainable energy system. J. Mater. Res. 34, 3030-3039 (2019) 
31. Gañán-Calvo, A. M., Rebollo-Munoz, N. \& Montanero, J. The minimum or natural rate of flow and droplet size ejected by Taylor cone-jets: physical symmetries and scaling laws. N. J. Phys. 15, 033035 (2013).

32. Rosell-Llompart, J., Grifoll, J. \& Loscertales, I. G. Electrosprays in the cone-jet mode: from Taylor cone formation to spray development. J. Aerosol Sci. 125, 2-31 (2018).

33. Fedorets, A. A. et al. Self-assembled levitating clusters of water droplets: pattern-formation and stability. Sci. Rep. 7, 1888 (2017).

34. Fialkowski, M. et al. Principles and implementations of dissipative (dynamic) self-assembly. J. Phys. Chem. B 110, 2482-2496 (2006).

35. Kashfipour, M. A., Mehra, N., Dent, R. S. \& Zhu, J. Regulating intermolecular chain interaction of biopolymer with natural polyol for flexible, optically transparent and thermally conductive hybrids. Eng. Sci. 8, 11-18 (2019).

36. Huang, $H$. et al. Tunable thermal-response shape memory bio-polymer hydrogels as body motion sensors. Eng. Sci. 9, 60-67 (2020).

37. Yao, X. et al. Adaptive fluid-infused porous films with tunable transparency and wettability. Nat. Mater. 12, 529-534 (2013).

38. Liu, H. et al. Stretchable conductive nonwoven fabrics with self-cleaning capability for tunable wearable strain sensor. Nano Energy 66, 104143 (2019).

39. Worsley, M. A. et al. Synthesis of graphene aerogel with high electrical conductivity. J. Am. Chem. Soc. 132, 14067-14069 (2010).

40. Tang, Z., Shen, S., Zhuang, J. \& Wang, X. Noble-metal-promoted threedimensional macroassembly of single-layered graphene oxide. Angew. Chem. Int. Ed. 122, 4707-4711 (2010).

41. Marinho, B., Ghislandi, M., Tkalya, E. \& Koning, C. E. Electrical conductivity of compacts of graphene, multi-wall carbon nanotubes, carbon black, and graphite powder. Powder Technol. 221, 351-358 (2012).

42. Naghdi, S., Rhee, K. Y. \& Park, S. J. A catalytic, catalyst-free, and roll-to-roll production of graphene via chemical vapor deposition: low temperature growth. Carbon 127, 1-12 (2018).

43. Cao, M. S., Wang, X. X., Cao, W. Q. \& Yuan, J. Ultrathin graphene: electrical properties and highly efficient electromagnetic interference shielding. J. Mater. Chem. C. 3, 6589-6599 (2015).

44. Sun, K. et al. Tunable negative permittivity in flexible graphene/PDMS metacomposites. J. Phys. Chem. C. 123, 23635-23642 (2019).

45. Xie, $\mathrm{P}$. et al. Tunneling-induced negative permittivity in $\mathrm{Ni} / \mathrm{MnO}$ nanocomposites by a bio-gel derived strategy. J. Mater. Chem. C. 8, 3029-3039 (2020).

46. $\mathrm{Li}, \mathrm{T}$. et al. Achieving better greenhouse effect than glass: visibly transparent and low emissivity metal-polymer hybrid metamaterials. ES Energy Environ. 5, 102-107 (2019).

47. Nel, A. Air pollution-related illness: effects of particles. Science 308, 804-806 (2005).

48. Mahowald, N. Aerosol indirect effect on biogeochemical cycles and climate. Science 334, 794-796 (2011).

49. Jerrett, M. Atmospheric science: the death toll from air-pollution sources. Nature 525, 330-331 (2015)

50. Yang, X., You, F., Zhao, Y., Bai, Y. \& Shao, L. Confinedly assembling surface nanocoating to manipulate nanofiltration membranes for highly-efficient dye removal. ES Energy Environ. 1, 106-113 (2018).

51. El-Kady, M. F., Strong, V., Dubin, S. \& Kaner, R. B. Laser scribing of highperformance and flexible graphene-based electrochemical capacitors. Science 335, 1326-1330 (2012).
52. Wei, H. et al. Advanced porous hierarchical activated carbon derived from agricultural wastes toward high performance supercapacitors. J. Alloy. Compd. 820, 153111 (2020).

53. Wei, H. et al. Multifunctions of polymer nanocomposites: environmental remediation, electromagnetic interference shielding, and sensing applications. ChemNanoMat 6, 174-184 (2020).

54. Chen, J. et al. An overview of stretchable strain sensors from conductive polymer nanocomposites. J. Mater. Chem. C. 7, 11710-11730 (2019).

\section{Acknowledgements}

This work was supported by the National Natural Science Foundation of China (Nos. 51873029, 51973028, 51925302, and 2191101289), the Innovation Program of Shanghai Municipal Education Commission (No. 2017-01-07-00-03-E00024), the Program of Shanghai Academic Research Leader (No. 18XD1400200), and Donghua University International Postdoctoral Program.

\section{Author contributions}

S.Z., H.L., and B.D. developed the research idea and design, and involved in data interpretation. S.Z., H.L., B.L., and J.Y. carried out the experiments and data analysis.

\section{Competing interests}

The authors declare no competing interests.

\section{Additional information}

Supplementary information is available for this paper at https://doi.org/10.1038/s41467020-18977-6.

Correspondence and requests for materials should be addressed to B.D.

Peer review information Nature Communications thanks the anonymous reviewers for their contributions to the peer review of this work

Reprints and permission information is available at http://www.nature.com/reprints

Publisher's note Springer Nature remains neutral with regard to jurisdictional claims in published maps and institutional affiliations.

Open Access This article is licensed under a Creative Commons Attribution 4.0 International License, which permits use, sharing, adaptation, distribution and reproduction in any medium or format, as long as you give appropriate credit to the original author(s) and the source, provide a link to the Creative Commons license, and indicate if changes were made. The images or other third party material in this article are included in the article's Creative Commons license, unless indicated otherwise in a credit line to the material. If material is not included in the article's Creative Commons license and your intended use is not permitted by statutory regulation or exceeds the permitted use, you will need to obtain permission directly from the copyright holder. To view a copy of this license, visit http://creativecommons.org/ licenses/by/4.0\%

(C) The Author(s) 2020 